\title{
Visualization of CAD and CAE Files using VTK and MFC
}

\author{
Mangesh Nayak \\ College of Computer \& Information Science, Srinivas University, Mangalore, India \\ E-mail: nayakmangesh@gmail.com
}

Type of the Paper: Research Paper.

Type of Review: Peer Reviewed.

Indexed in: OpenAIRE.

DOI: http://doi.org/10.5281/zenodo.1163159.

Google Scholar Citation: IJMTS

\section{How to Cite this Paper:}

Nayak, Mangesh. (2018). Visualization of CAD and CAE Files using VTK and MFC. International Journal of Management, Technology, and Social Sciences (IJMTS), 3(1), 18-24. DOI: http://doi.org/10.5281/zenodo.1163159.

International Journal of Management, Technology, and Social Sciences (IJMTS)

A Refereed International Journal of Srinivas University, India.

(C) With Authors.

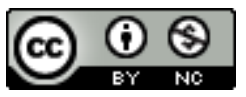

This work is licensed under a Creative Commons Attribution-Non Commercial 4.0 International License subject to proper citation to the publication source of the work.

Disclaimer: The scholarly papers as reviewed and published by the Srinivas Publications (S.P.), India are the views and opinions of their respective authors and are not the views or opinions of the SP. The SP disclaims of any harm or loss caused due to the published content to any party. 


\title{
Visualization of CAD and CAE Files using VTK and MFC
}

\author{
Mangesh Nayak \\ College of Computer \& Information Science, Srinivas University, Mangalore, India \\ E-mail: nayakmangesh@gmail.com
}

\begin{abstract}
This software is capable of reading the file formats like - .OBJ, .WRL, .STL, .STEP, .IGES and 3D scenes can be rendered with an inclusion of the properties viz. applying variousLights, Material Color, and Options for Solid, Wire frame, Points and Transparency viewing, Texture Mapping, Transformation, Walkthrough, Cut Sections, Background color selection, Background and Base image selection, Markup features such as Sealing and Measurements, Different camera views, Parts selection and deletion, Copying the rendered object to the clipboard and enabling to view more than one models through multiple tabbed windows.Stereovision feature has been added to this software, which gives better 3D visual effect at lower cost. This software is also intended to incorporate ease of use and portability so that it can run on any low end workstation having windows operating system.
\end{abstract}

Keywords: VTK (Visualization Toolkit), Kitware, CAE Visualization, CAD, ITK.

\section{INTRODUCTION :}

Aeronautical engineering, mechanical engineering, electronics and electrical engineering, computer science, instrumentation, library science and management, foundry, investment casting, rapid prototyping, CNC shop floors, $\mathrm{CAD} / \mathrm{CAM} / \mathrm{CAE}$, test rigs etc. are the major facilities available in Computer Research Organisations like IT companies, Research Companies etc [1].

$\mathrm{CAD} / \mathrm{CAM} / \mathrm{CAE}$ centre is the centralized facility for Computer Aided Design, Computer Aided Manufacturing and Computer Aided Engineering activities in some of the Computer Research Organisation, where the 3D modelling components and major assemblies are modelled and assembled using various 3D modelling software. These models are being extensively used for design, finite element analysis, generating CNC part programs etc [2].

The 3D models software are based on NURBS (Non Uniform Rational BSpline). The modeller not only holds the geometric information, but also topological information of the object. Since these packages define the object in more extensive way, the model file size will be bigger, hence requires powerful computer system.

$\mathrm{CAD} / \mathrm{CAM} / \mathrm{CAE}$ centre on its way to a state of art virtual reality lab; virtual reality the name itself says that, adding the sense of realism to a computer generated visualization of each components and assemblies, where user should be able to do transformation, walkthrough and so many other manipulations on the components/assemblies and to take decisions pertaining to design modifications, assembly procedure, etc., which otherwise would have been a costly and time consuming affair in real world situation [3].

Survey reveals that, no visualization software available for the engineering application, which uses CAE model and provides stereo vision features. Therefore it is required to generate stereo view is one of the method to add realism to computer generated scene, where and three dimensional scene can be viewed using stereo glass to perceive 3D depth or 3D effect. Enabling object visualization in 3D space with immersion is essence of virtual reality. Therefore, an attempt has made in this dissertation to develop "Stereo Visualisation of 
Cad and CAE files”, here after called as TECHVISION [4].

The software supports visualization of different CAE models and enables user to open and render various file formats generated by the different software used in CAE Research Organisation. User can carry out various operations based on the models that render using the application. The software supports stereovision, using which one can have real feel of the virtual components and assemblies, even before the actual product is manufactured. This software is also intended to incorporated ease of use and portability, so that it can run on any low end workstation having windows operating system.

\section{OVERVIEW :}

The CAE software package have a capability to export its components and sub-assemblies and assemblies into sophisticated software generated ASCII format files like OBJ, .WRL, .STL, .STEP, .IGES etc. STEP "Stereo Integrated Environment in Passive Mode" have a capability to browse required model files OBJ, .WRL, .STL, .STEP, .IGES read the selected files and save model information of the $3 \mathrm{D}$ model in some data structures and finally render the 3D object model using that data structure. The software has developed incorporating following characteristics.

- Simplicity : for ease of use and user friendliness.

- Consistency : to operate in a consistent and predictable way

- Completeness : no inconvenient omissions in viewing functions.

- Robustness : tolerate against minor instances of misuse.

- Performance : efficient programming for faster response.

- Economy : low cost of development and implementation.

The primary objective of this software is to provide the client with the fully functional virtual reality system that allows them to explore their models or to develop prototypes of a virtual environment for later use in larger systems without having to use more expensive resources during the development process.

\section{TOOLS ANDARCHITECTURE :}

The Visualization Toolkit (VTK) is an opensource, freely available software system for 3D computer graphics, image processing, and visualization. VTK consists of a $\mathrm{C}++$ class library and several interpreted interface layers including Tcl/Tk, Java, and Python. Kitware, whose team created and continues to extend the toolkit, offers professional support and consulting services for VTK. VTK supports a wide variety of visualization algorithms including scalar, vector, tensor, texture, and volumetric methods; and advanced modeling techniques such as implicit modeling, polygon reduction, mesh smoothing, cutting, contouring, and Delaunay triangulation. VTK has an extensive information visualization framework, has a suite of 3D interaction widgets, supports parallel processing, and integrates with various databases on GUI toolkits such as Qt and Tk [8]. VTK is cross-platform and runs on Linux, Windows, and Mac and UNIX platforms. VTK also includes ancillary support for 3D interaction widgets, two and three-dimensional annotation, and parallel computing. At its core VTK is implemented as a $\mathrm{C}++$ toolkit, requiring users to build applications by combining various objects into an application. The system also supports automated wrapping of the $\mathrm{C}++$ core into Python, Java, and Tcl, so that VTK applications may also be written using these interpreted programming languages [5].

VTK is used worldwide in commercial applications, research, and development, and is the basis of many advanced visualization applications such as Molekel, ParaView, VisIt, VisTrails, MOOSE, 3DSlicer, MayaVi, and OsiriX. VTK is an open-source toolkit licensed under the BSD license [6-7]. 


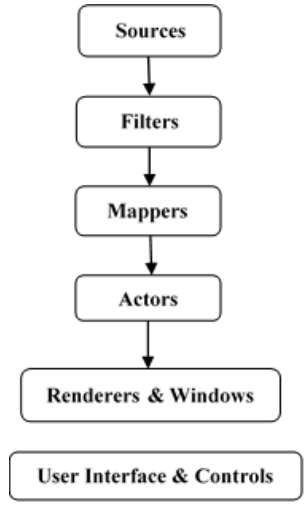

Figure 1:VTK Pipeline Architecture [5-6]

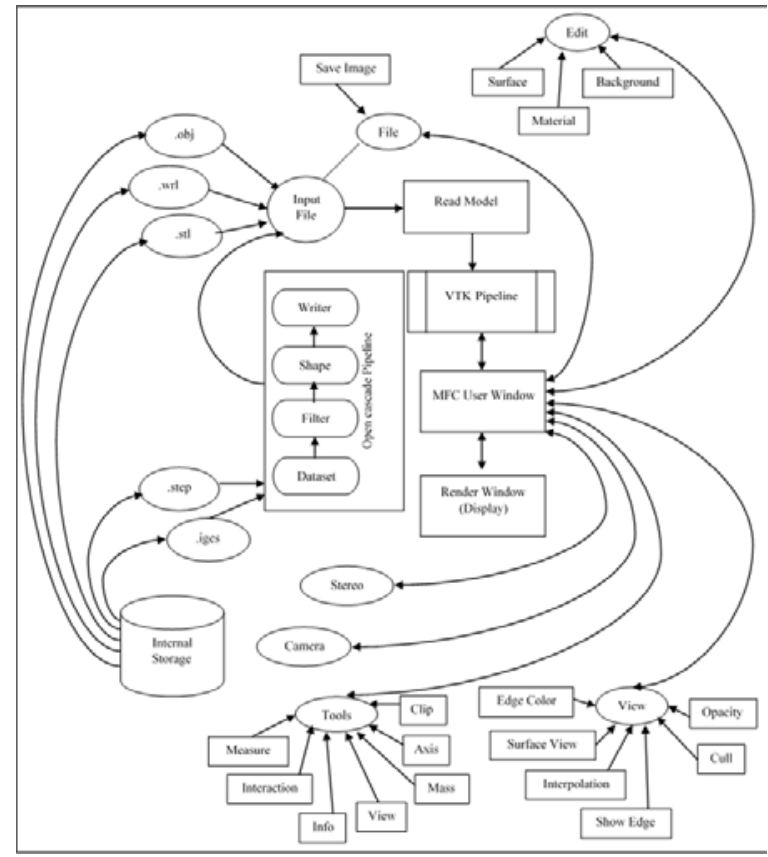

Figure 2: Complete Architecture of Tech-Vision

\section{TESTING :}

Specific knowledge of the application's code/internal structure and programming knowledge in general is not required. The tester is aware of what the software is supposed to do but is not aware of how it does it. For instance, the tester is aware that a particular input returns a certain, invariable output but is not aware of how the software produces the output in the first place.

Software is tested from two different perspectives.

- Internal program logic is exercised using "White Box" test case design techniques.
Using white-box testing, test cases are derived that

0 assuring that all independent and dependent paths within a module have been exercised at least once.

o Examine every logical decisions on their true and false sides.

o Examine all loops at their boundaries and within their operational bounds/criteria.

o Examine internal data structure to ensure their validity.

- $\quad$ Software requirement are examined using "Black Box" test techniques. The test focuses on the functional requirements of the software. Black-box testing is not an alternative to white-box testing. Rather, it is a complementary approach that is likely to uncover a different class of errors than whitebox methods. Tests are designed to answer the following questions:

o How is functional validity used?

o How is system behaviour and performance tested?

o What classes of input will make good test cases?

0 Is the system particularly sensitive to certain input values?

o How are the boundaries of a data class isolated?

o What data rates and data volume can the system tolerate?

o What effect will specific combinations of data have on system operation?

In both cases, the intention is to find the maximum number of errors with the minimum amount of effort and time. A set of test cases designed to examine both internal logic and external requirement is designed and documented, expected results are defined and actual results are recorded [8-13].

\section{SCOPE FOR FURTHER WORK :}

Tech-Vision has been developed using VC++ MFC Application Wizard and Visualization Toolkit with open CASCADE displaying the models using VTK and tools for Graphical User Interface (GUI) are used from ApplicationWizard and MFC (Microsoft Foundational 
Classes) of Visual C++ 2010 [14].

The additional features and further improvement that have to be done are mentioned below:

- Visualization Toolkit (VTK) is open source software which is an extremely handy tool. Whereas there are no experienced tutorials available on the internet for study. So we need to work and implement for future reference.

- VTK can be used to optimize the scene graph and other capabilities.

- VTK supports various kinds file format reading. But reading any file format is not possible. Only some kinds which are supported by VTK is possible.

- In order to get a high featured software, we need to combine various open source visual software like OpenAL, OpenCV, Open CASCADE Technology etc. when combining we can extract more features.

- In VTK object explode feature is not available. Object explode means a rendering object must dismantle and again join. This feature is highly recommended for visualization software.

- Assembly and disassembly can be done using a mouse, assembly and disassembly sequence can be stored as a movie file, and this movie file can be taken anywhere to view assembly and disassembly as a movie. Hence new software should incorporate the feature of assembly and disassembly and a record button to record the assembly and disassembly sequence. This record button should also capable to record all type of transformations and walkthrough.

- There is no provision for Mass, Center of Gravity Calculation in VTK.

- Virtual piping can be done in the future work, as VTK does not support this feature.

- Additional Markup features can be included for the present VTK supported Markup features.

- Realistic effect can be given to the rendered model by giving sound effects in the future work that will be carried out.

- In VTK, Copy option will allow to copying the rendered model onto the clipboard. The user himself has to open any of the paint or editing applications and paste the copied image. So this work can be extended in which the user should be allowed to enter the filename in software application itself, in any of the picture file formats. This should thereby save that copied file in whichever location the user requires.

- Developed software reads only the tessellated data. So it can be extended to read other data.

- Work can be carried out to bring out the Reflection and Shadow feature, as it is not supported in developed software.

- Collision detection can be incorporated into the future work.

- Developed software should run on any platform by Making it platform independent can further enhance this software.

- Additional features like Interfacing with other VR devices such as Hand Gloves, HMD, and wearable VR Suits etc.

Most important that VTK which we developed using MFC, but it can be effectively implemented by QT, Python, .NET, etc.[15-20

\section{CONCLUSION :}

An attempt was made to come out with an actual tool. For this, a detailed study with the requirement was done. Hence this project is divided into two different modules. All requirements for each module were taken. The user interfaces are being created in an attractive manner following the company standards. User manual developed guides any user to use the tools without any difficulties. Scaling the actual requirement of the system develops the software. For this first, we need to understand the system design and the system should be divided into subsystems. Later these subsystems are developed and tested. The tested subsystems are put together to get the actual system.

However, we combined the different software in order to get the maximum feature and high end output for the results. In MFC developer can use its feature to develop an high-end software application using its inbuilt features like file browsing, buttons, icons, resources, tools etc., but in Visualization Toolkit is used as backend application because the application which we 
developed must require both MFC and VTK in order to function appropriately when it reaches in the hands of client or user. The developed software will interact with the user and provide ultimate visualization product which a client needed the most.

\section{ACKNOWLEDGMENT :}

I sincere thanks towards my external guide $\mathrm{Mr}$. K. Vijayananda, Scientist 'F', GAS TURBINE RESEARCH ESTABLISHMENT, DRDO, Bengaluru and to my internal guide Dr. Manjaiah D.H., Professor, Department of Computer Science, Mangalore University and to Mr. AJAY KUMAR G, Research Scholar, Virtual Environmental Lab, GSAIM, ChungAngUniversity, Heukseok dong, Dongjak-gu, Seoul, South Korea. His valuable guidance and support in carrying this project.

\section{REFERENCES :}

[1] Ma, S. Q., \& Gao, Z. L. (2009, May). A solution to integrate CAD/CAE system and VR for complex product design processes. In Information and Computing Science, 2009. ICIC'09. Second International Conference on (Vol. 4, pp. 331-333). IEEE.

[2] Brunetti, G., \& Golob, B. (2000). A featurebased approach towards an integrated product model including conceptual design information. Computer-Aided Design, 32(14), 877-887.

[3] Bimber, O., Encarnacao, L. M., \& Stork, A. (2000). A multi-layered architecture for sketch-based interaction within virtual environments. Computers \& Graphics, 24(6), 851-867.

[4] Schroeder, W. J., Martin, K. M., \& Lorensen, W. E. (1996, October). The design and implementation of an objectoriented toolkit for 3D graphics and visualization. In Proceedings of the 7th conference on Visualization'96 (pp. 93-ff). IEEE Computer Society Press.

[5] Avila, L. S. (2004). The VTK user's guide: updated for VTK version 4.4; [install, use and extend the visualization toolkit; covers installation on PC, Unix and Mac OSX; includes example scripts, $\mathrm{C}++$ source code, images and data; shows how to extend VTK for your own applications]. Kitware.

[6] Lingaraju11, G., Bagewadi, C., \& Nithiarasu, P. (2008). Real Time Scientific Visualization of the ALE Based Free Surface Simulation for Virtual Reality Applications. IJCSNS, 8(9), 399.

[7] Pieper, S., Lorensen, B., Schroeder, W., \& Kikinis, R. (2006, April). The NA-MIC Kit: ITK, VTK, pipelines, grids and 3D slicer as an open platform for the medical image computing community. In Biomedical Imaging: Nano to Macro, 2006. 3rd IEEE International Symposium on (pp. 698-701). IEEE.

[8] Fabian, N., Moreland, K., Thompson, D., Bauer, A. C., Marion, P., Gevecik, B., ...\& Jansen, K. E. (2011, October). The preview coprocessing library: A scalable, general purpose in situ visualization library. In Large Data Analysis and Visualization (LDAV), 2011 IEEE Symposium on (pp. 89-96). IEEE.

[9] Ahrens, J., Geveci, B., Law, C., Hansen, C., \& Johnson, C. (2005). 36-paraview: An end-user tool for large-data visualization. The visualization handbook, 717.

[10] Heer, J., Card, S. K., \&Landay, J. A. (2005, April). Prefuse: a toolkit for interactive information visualization. In Proceedings of the SIGCHI conference on Human factors in computing systems (pp. 421-430). ACM.

[11] Schroeder, W. J., Avila, L. S., \& Hoffman, W. (2000). Visualizing with VTK: a tutorial. IEEE Computer graphics and applications, 20(5), 20-27.

[12] Pieper, S., Halle, M., \&Kikinis, R. (2004, April). 3D Slicer. In Biomedical Imaging: Nano to Macro, 2004. IEEE International Symposium on (pp. 632-635).

[13] Taka, S. J., \&Srinivasan, S. (2011). NIRViz: 3D visualization software for multimodality optical imaging using Visualization Toolkit (VTK) and Insight Segmentation Toolkit (ITK). Journal of 
digital imaging, 24(6), 1103-1111.

[14] Schildt Herbert, (2008). MFC Programming from the ground up, Tata McGraw hill publications.

[15] Taka, S. J., \&Srinivasan, S. (2011). NIRViz: 3D visualization software for multimodality optical imaging using Visualization Toolkit (VTK) and Insight Segmentation Toolkit (ITK). Journal of digital imaging, 24(6), 1103-1111.

[16] Buriol, T. M., \&Scheer, S. (2008). CAD and $\mathrm{CAE}$ integration through scientific visualization techniques for illumination design. Tsinghua Science \& Technology, 13, 26-33.

[17] Qu, X., \&Stucker, B. (2003). A 3D surface offset method for STL-format models. Rapid Prototyping Journal, 9(3), 133-141.

[18] Honjo, T., \& Lim, E. M. (2001). Visualization of landscape by VRML system. Landscape and urban planning, 55(3), 175-183.

[19] Pieper, S., Lorensen, B., Schroeder, W., \&Kikinis, R. (2006, April). The NA-MIC Kit: ITK, VTK, pipelines, grids and 3D slicer as an open platform for the medical image computing community. In Biomedical Imaging: Nano to Macro, 2006. 3rd IEEE International Symposium on (pp. 698-701). IEEE.

[20] Wee, L. K., Chai, H. Y., \&Supriyanto, E. (2011). Surface rendering of three dimensional ultrasound images using VTK. NISCAIR-CSIR, India, JSIR 70(06), 421426. 